\title{
COMUNICAÇÃo BREve Inventário de Depressão de Beck (BDI) e Escala de Avaliação de Depressão de Hamilton (HAM-D) em pacientes com epilepsia
}

\author{
Beck Depression Inventory (BDI) and Hamilton Rating Scale \\ for Depression (HAM-D) in patients with epilepsy \\ Guilherme Nogueira M. de Oliveira, , Gerardo Maria de Araujo Filho3,4, Arthur Kummer', João Vinícius \\ Salgado ${ }^{1}$, Eduardo Jardel Portela ${ }^{2,5}$, Sílvio Roberto Sousa-Pereira ${ }^{5}$, Antônio Lucio Teixeira
}

\section{RESUMO}

Objetivo: Determinar os pontos de melhor sensibilidade e especificidade do Inventário de Depressão de Beck (BDI) e da Escala de Avaliação de Depressão de Hamilton (HAM-D) no diagnóstico de depressão associada à epilepsia. Métodos: Setenta e três pacientes de um centro de referência no tratamento da epilepsia foram submetidos à avaliação neuropsiquiátrica. Foram colhidos dados clínicos e sociodemográficos, sendo utilizados os seguintes instrumentos: entrevista clínica estruturada (MINI-PLUS) para diagnóstico psiquiátrico conforme o DSM-IV, HAM-D e BDI. Resultados: No momento da entrevista, 27,4\% dos pacientes estavam deprimidos e $37 \%$ preenchiam critérios para diagnóstico de depressão maior ao longo da vida. A análise da curva ROC indicou que o ponto de corte em 16 (> 16) para o BDI

\section{Palavras-chave}

Epilepsia, depressão, diagnóstico, Escala de Avaliação de Depressão de Hamilton, Inventário de Depressão de Beck. (sensibilidade de 94,4\%, especificidade de 90,6\%) e em 16 (> 16) para a HAM-D (sensibilidade de $95 \%$, especificidade de 75,5\%) representou dicotomização ótima entre deprimidos e não deprimidos. Ambos os instrumentos apresentaram um valor preditivo negativo superior a 95\%. Conclusão: A frequência de depressão maior é elevada em pacientes com epilepsia. BDI e a HAM-D podem auxiliar o clínico na identificação da depressão associada à epilepsia, diminuindo seu subdiagnóstico.

\begin{abstract}
Objective: To determine cutoff points of highest sensitivity and specificity on the Beck Depression Inventory (BDI) and Hamilton Depression Rating Scale (HAM-D) for depression diagnosis in epilepsy. Methods: Seventy-three patients from a referral center for the treatment of epilepsy underwent neuropsychiatric evaluation. We collected clinical and socio-demographic data, and applied the following instruments: Structured Clinical Interview (MINI-PLUS) for psychiatric diagnosis according to DSM-IV, HAM-D and BDI. Results: At assessment, 27.4\% of the patients were depressed and 37\% met diagnostic criteria for lifetime major depression. The ROC curve analysis indicated that a score $>16$ on the BDI ( $94.4 \%$ sensitivity, $90.6 \%$ specificity) and $>16$ on
\end{abstract}

1 Universidade Federal de Minas Gerais (UFMG), Serviço de Neurologia, Hospital das Clínicas, Unidade de Neuropsiquiatria. 2 Hospital Felício Rocho, Núcleo Avançado de Tratamento das Epilepsias (NATE), Belo Horizonte. 3 Universidade Federal de São Paulo (Unifesp), Departamento de Neurologia e Neurocirurgia. 


\section{Keywords}

Epilepsy, depression,

diagnosis, Hamilton

Depression Rating Scale,

Beck Depression Inventory. the HAM-D (95\% sensitivity, 75.5\% specificity) revealed great dichotomy between depressed and nondepressed patients. Both instruments showed a negative predictive value exceeding 95\%. Conclusion: The frequency of major depression is elevated in patients with epilepsy. BDI and HAM-D can help physicians in the identification of depression in epilepsy, reducing its underdiagnosis.

\section{INTRODUÇÃO}

A depressão é a comorbidade psiquiátrica mais comum nas síndromes epilépticas, com prevalência ao longo da vida de $17 \%$ em indivíduos com epilepsia na comunidade1. No entanto, a depressão frequentemente não é diagnosticada e muitos pacientes permanecem sem tratamento adequado ${ }^{2,3}$.

Escalas e inventários de depressão são utilizados para quantificar sintomas depressivos e podem auxiliar no diagnóstico a partir de pontos de corte específicos ${ }^{4,5}$. O Inventário de Depressão de Beck (BDI) ${ }^{4}$ e a Escala de Depressão de Hamilton (HAM-D) $)^{5}$ têm sido utilizados na avaliação de sintomas depressivos na epilepsia ${ }^{3,6}$, mas ainda não há publicações brasileiras que tenham descrito os pontos de corte desses instrumentos em indivíduos nessa população. Portanto, realizamos um estudo transversal para avaliar a aplicação desses instrumentos em um grupo de pacientes com epilepsia.

\section{MÉTODOS}

Foi realizado um estudo transversal em pacientes com epilepsia do lobo temporal (ELT) acompanhados no Ambulatório de Epilepsias do Serviço de Neurologia do Hospital das Clínicas da Universidade Federal de Minas Gerais (UFMG), que é referência no estado para o tratamento de epilepsia. Foram excluídos os pacientes menores de 18 anos; submetidos a neurocirurgias prévias e com outras doenças neurológicas (delirium, demência e acidente vascular encefálico).

\section{Avaliação neuropsiquiátrica}

O Mini International Neuropsychiatric Interview (MINI-Plus) ${ }^{7}$ foi utilizado neste trabalho para definir o diagnóstico de episódio depressivo maior atual segundo os critérios do DSM-IV. $\mathrm{Na}$ caracterização dos sintomas depressivos, foi utilizado o $\mathrm{BDI}$, que possui 21 itens, com pontuação total de 0 a $63^{4}$. A HAM-D é o instrumento de avaliação de sintomas depressivos mais utilizado no mundo ${ }^{5}$. Neste estudo, optou-se pela versão da HAM-D com 24 itens, cujo escore máximo é 76, visto que essa versão atenua o peso dos sintomas somáticos, aumentando sua especificidade.

\section{Análise estatística}

Foi investigada a existência de correlação entre os instrumentos para avaliar gravidade de sintomas depressivos (BDI e
HAM-D) por meio do coeficiente de correlação de Spearman. O escore ótimo de maior sensibilidade ou especificidade do BDI e da HAM-D para diagnóstico de depressão foi calculado por meio da receiver operating characteristic curve (curva ROC).

Todas as análises foram realizadas utilizando-se o programa estatístico SPSS versão 15.0 para Windows. O programa MedCalc 8.0 foi utilizado para análise da curva ROC. Um valor de p bilateral menor que 0,05 foi adotado como nível de significância estatística para todos os testes.

\section{RESULTADOS}

Setenta e três pacientes de ambos os gêneros ( $53 \%$ do sexo feminino) com ELT foram submetidos à avaliação neuropsiquiátrica. A população estudada foi composta por pacientes com idade média (desvio-padrão - DP) de 42,2 (10,0) anos, com crises epilépticas iniciadas na infância, média (DP) de 8,5 $(9,8)$ anos de idade, de longo tempo de evolução, média (DP) de 33,7 $(12,2)$ anos, e com frequência média (DP) de $4,8(7,6)$ crises ao mês. A escolaridade média (DP) foi de $6,9(3,5)$ anos de estudo.

Mais de um terço dos pacientes (37\%) recebeu o diagnóstico de transtorno depressivo maior durante a vida, e 27,4\% dos pacientes estavam deprimidos no momento da avaliação. Informações mais detalhadas sobre as características clínicas, sociodemográficas e frequência de transtornos psiquiátricos dessa amostra estão descritas em outra publicação?.

A análise da curva ROC indica que o ponto de corte do BDI em 16 (> 16) representa dicotomização ótima entre deprimidos (episódio depressivo maior atual) e não deprimidos (sensibilidade de $94,4 \%$, especificidade de $90,6 \%$, valor preditivo positivo de $79,1 \%$ e valor preditivo negativo de $97,7 \%$ ). A área sob a curva foi de 0,963 (intervalo de confiança de 95\%: 0,888-0,993), indicando boa propriedade discriminativa do instrumento, conforme demonstrado pela figura 1.

O ponto de corte da HAM-D em 16 (> 16), mediante a análise da curva ROC, permite a melhor discriminação entre deprimidos (episódio depressivo maior atual) e não deprimidos (sensibilidade de $95 \%$, especificidade de $75,5 \%$, valor preditivo positivo de 59,4\% e valor preditivo negativo de 97,6\%). A área sob a curva foi de 0,907 (intervalo de confiança de 95\%: 0,816-0,962), indicando adequada propriedade discriminativa do instrumento, conforme apresentado pela figura 2. Houve correlação positiva entre os instrumentos BDI e HAM-D ( $\rho$ de Spearman $=0,835 ; p<0,001$ ). 


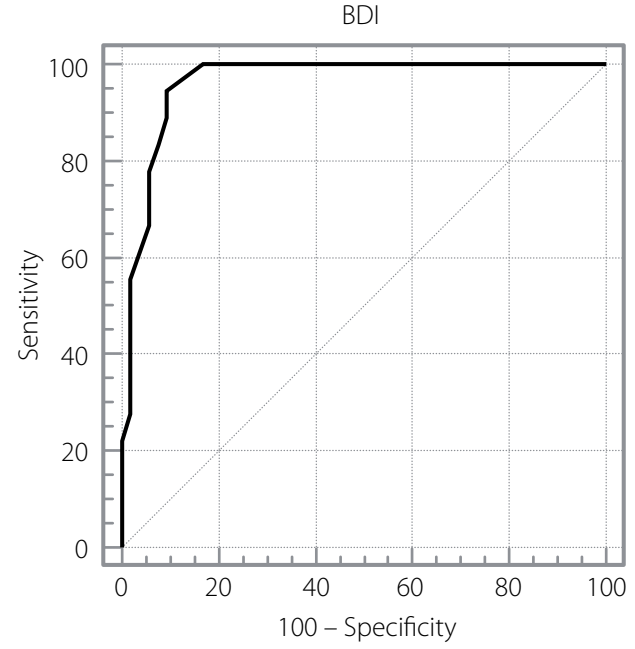

Figura 1. Receiver operating characteristic curve (curva ROC) do $\mathrm{BDI}$, com o ponto de corte ótimo assinalado (> 16).

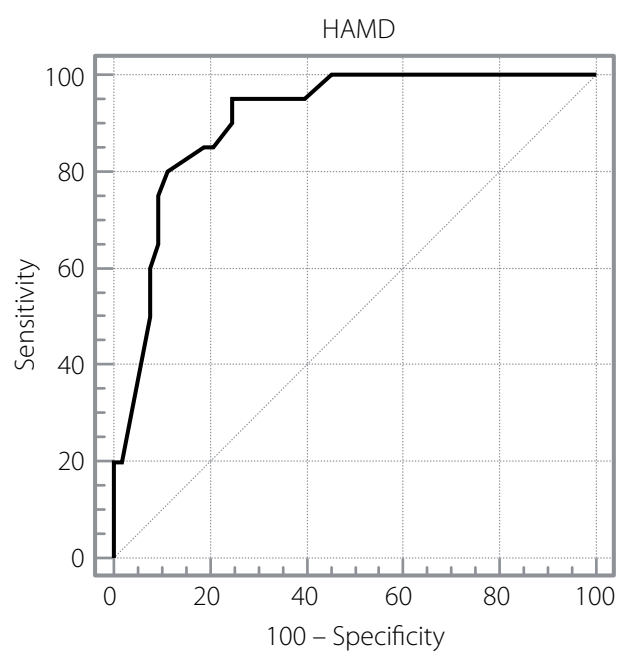

Figura 2. Receiver operating characteristic curve (curva $\mathrm{ROC}$ ) da HAM-D, com o ponto de corte ótimo assinalado (> 16).

\section{DISCUSSÃO}

O presente estudo contribui para facilitar o reconhecimento de depressão nas epilepsias, demonstrando a aplicabilidade clínica de instrumentos de avaliação de sintomas depressivos (BDI e HAM-D) na ELT.

$\mathrm{O}$ BDI foi o instrumento com maior sensibilidade e valor preditivo positivo no rastreamento da depressão em comparação à HAM-D. No entanto, pacientes com prejuízo cognitivo ou baixa escolaridade apresentam dificuldades no preenchimento de inventários como o BDI. Nesses casos, a HAM-D pode ser aplicada por entrevistador experiente no exame psiquiátrico e familiarizado com a entrevista de pacientes deprimidos ${ }^{5}$. No entanto, o fato de a HAM-D ser examinador-dependente pode limitar seu uso na prática clínica. Nesse contexto, é oportuno destacar a recente validação para o português de um instrumento de triagem da depres- são na epilepsia, o Inventário de Depressão em Transtornos Neurológicos para a Epilepsia (IDTN-E) ${ }^{8}$. Esse instrumento foi desenvolvido especificamente para a depressão na epilepsia e leva apenas 3 minutos ${ }^{9,10}$, podendo ser útil na triagem de depressão na qual não se tenha disponível um profissional capacitado na aplicação da HAM-D ou caso o paciente apresente dificuldades no preenchimento do BDI.

Estudos prévios foram realizados com esses e outros instrumentos de avaliação psicopatológica ${ }^{3,6,8,11}$. Por exemplo, Botega et al. aplicaram a Escala Hospitalar de Ansiedade e Depressão (HAD) em 56 pacientes com epilepsia e observaram que uma pontuação $>7$ na subescala que avalia depressão (HAD-D) proporcionou sensibilidade de 85,7\% e especificidade de $72,4 \%$ no reconhecimento dos quadros depressivos de acordo com o DSM-III-R ${ }^{11}$. Jones et al. estudaram as propriedades psicométricas do BDI-II em 174 pessoas com epilepsia, encontrando uma estrutura homogênea revelada pela elevada consistência interna (alpha de Cronbach de 0,94$)^{3}$. Apenas cinco itens apresentaram correlação item-total <0,60, e as correlações item-total variaram de 0,45 (pensamentos suicidas) a 0,78 (menos-valia). Esse estudo utilizou duas entrevistas psiquiátricas estruturadas, o MINI e a Entrevista Clínica Estrutura para DSM-IV (SCID), para o diagnóstico da depressão. Na análise da curva ROC, esses autores sugerem pontuações no BDI-II > 11 (ao utilizarem o MINI) e $>15$ (ao utilizarem o SCID) no rastreamento da depressão na epilepsia, demonstrando que diferentes pontos de corte podem ser obtidos se o objetivo for maior sensibilidade ou especificidade do instrumento ${ }^{3}$.

Os pontos de corte sugeridos pelo presente estudo (BDI $>16$ e HAM-D > 16) para discriminação entre indivíduos deprimidos e eutímicos são dados originais na literatura brasileira em relação ao diagnóstico de depressão na epilepsia. Esses pontos de corte são superiores àqueles propostos para a população geral ${ }^{3-5}$. Isso pode ser, ao menos em parte, atribuído ao fato de os sintomas depressivos em pacientes com epilepsia serem frequentemente confundidos com os efeitos adversos das drogas antiepilépticas e queixas cognitivas associadas à própria epilepsia, elevando os pontos de corte para uma maior especificidade ${ }^{12}$.

O presente estudo descreve a observação transversal de sintomas depressivos em indivíduos com ELT atendidos em um serviço de atenção terciária. Como a presente população é composta por pacientes em sua maioria com crises de difícil controle, esses dados podem ser importantes para serviços especializados, como em programas de cirurgia da ELT. De fato, parece haver uma associação entre o acometimento de estruturas temporais e os quadros depressivos. Essa ideia é reforçada por recente estudo espanhol que avaliou mais de 300 pacientes com crises parciais complexas. Os autores verificaram que os pacientes com ELT apresentaram maior frequência de depressão no último ano $(14,6 \%)$ em comparação àqueles com foco extratemporal $(6,5 \%)^{13}$. 
Por outro lado, como a amostra do presente trabalho representa uma subpopulação de pacientes com epilepsia grave, isso provavelmente limita a generalização dos dados para outros tipos de epilepsia e mesmo para pacientes com crises epilépticas na comunidade. Isso porque a sensibilidade dos instrumentos pode ser elevada em populações com transtornos prevalentes, como é o caso da depressão nessa amostra de pacientes com ELT. Além disso, apesar de utilizados na prática clínica e em pesquisas, a HAM-D e o BDI não foram desenvolvidos originalmente como instrumentos de rastreamento ou diagnóstico, mas sim como ferramentas de quantificação de sintomas depressivos em pacientes com depressão ${ }^{4,5}$. Portanto, é importante ressaltar que instrumentos de rastreamento não substituem o exame psiquiátrico. Nesse sentido, o trabalho de psiquiatras e neurologistas deve ser integrado para que o diagnóstico e o tratamento da depressão sejam corretamente instituídos, observando-se as particularidades da epilepsia ${ }^{14}$.

Os transtornos mentais na epilepsia devem ser observados de maneira longitudinal, atentando-se para a correlação temporal entre eles e as crises epilépticas ${ }^{15,12}$. Isso porque alterações de humor no período perictal são muito comuns e autolimitadas, com resposta pobre ao uso de fármacos antidepressivos $^{16}$. Todos os pacientes aqui avaliados apresentavam-se deprimidos no período interictal. Portanto, é recomendado que, juntamente à aplicação dos instrumentos, sejam também colhidos dados sobre a duração dos sintomas e sua associação com eventos ictais.

\section{CONCLUSÃO}

O BDI e a HAM-D mostraram desempenho satisfatório e podem ser utilizados em pesquisas e também na prática clínica na identificação dos indivíduos com depressão na epilepsia.

\section{AGRADECIMENTOS}

Agradecemos ao Dr. Renato Marchetti, pelas precisas observações na interpretação dos resultados deste trabalho. GuiIherme Nogueira M. de Oliveira foi bolsista da Coordenação de Aperfeiçoamento de Pessoal de Nível Superior (CAPES) e o trabalho foi financiado em parte pelo Edital Fapemig/Fapesp Neurociências - Epilepsia.

\section{REFERÊNCIAS}

1. Tellez-Zenteno JF, Patten SB, Jetté N, Williams J, Wiebe S. Psychiatric comorbidity in epilepsy: a population-based analysis. Epilepsia. 2007;48(12):2336-44.

2. De Oliveira GNM, Kummer A, Salgado JV, Portela EJ, Sousa-Pereira SR, David AS, et al. Psychiatric disorders in temporal lobe epilepsy: an overview from a tertiary service in Brazil. Seizure. 2010;19(8):479-84.

3. Jones JE, Hermann BP, Woodard JL, Barry JJ, Gilliam F, Kanner AM, et al. Screening for major depression in epilepsy with common self-report depression inventories. Epilepsia. 2005;46(5):731-5.

4. Gorestein C, Andrade L. Beck Depression Inventory: psychiometric properties of the Portuguese version. Rev Psiq Clín. 1998;25:245-50.

5. Moreno RA, Moreno DH. Hamilton (HAM-D) and Montgomery \& Asberg (MADRS) rating scales. Rev Psiq Clín. 1998;25(5):262-72.

6. Krishnamoorthy ES. The evaluation of behavioral disturbances in epilepsy. Epilepsia. 2006;47(s2):3-8

7. Amorim P. Mini International Neuropsychiatric Interview (MINI): validation of a short structured diagnostic psychiatric interview. Rev Bras Psiquiatr. 2000;22(3):106-15.

8. De Oliveira GNM, Kummer A, Salgado JV, Portela EJ, Sousa-Pereira SR, David AS, et al. Brazilian version of the Neurological Disorders Depression Inventory for Epilepsy (NDDI-E). Epilepsy Behav. 2010;19(3):328-31.

9. Gilliam FG, Barry JJ, Hermann BP, Meador KJ, Vahle V, Kanner AM. Rapid detection of major depression in epilepsy: a multicentre study. Lancet Neurol. 2006;5(5):399-405.

10. Friedman DE, Kung DH, Laowattana S, Kass JS, Hrachovy RA, Levin HS. Identifying depression in epilepsy in a busy clinical setting is enhanced with systematic screening. Seizure. 2009;18(6):429-33

11. Botega NJ, Pondé MP, Medeiros P, Lima MG, Guerreiro CAM. Validação da escala hospitalar de ansiedade e depressão (HAD) em pacientes epiléticos ambulatoriais. J Bras Psiquiatr. 1998;47(6):285-9.

12. Araújo Filho GM, Pellegrino RV, Yacubian EMT. Psychiatric disorders in epilepsy: a proposal for classification by the ILAE commission on neuropsychiatry. J Epilepsy Clin Neurophysiol. 2008;14(3):119-23.

13. Sanchez-Gistau V, Pintor L, Sugranyes G, Baillés E, Carreño M, Donaire A, et al. Prevalence of interictal psychiatric disorders in patients with refractory temporal and extratemporal lobe epilepsy in Spain: a comparative study. Epilepsia. 2010;51(7):1309-13.

14. Kanner AM. When did neurologists and psychiatrists stop talking to each other? Epilepsy Behav. 2003;4(6):597-601.

15. Kanner AM. Depression and epilepsy: a new perspective on two closely related disorders. Epilepsy Curr. 2006;6(5):141-6.

16. Mellers JDC. Epilepsy. In: Lishman's Organic psychiatry: a textbook of neuropsychiatry. Wiley-Blackwell; 2009. 